\title{
P-Glycoprotein Inhibitors from Natural Sources as Chemopreventives
}

\section{Pankaj Gupta*}

School of Medical and Allied Sciences, KR Mangalam University, Sohna Road, Gurgaon, Haryana, India

P-glycoprotein (P-gp) is a transmembrane permeability glycoprotein belonging to the ATP binding cassette (ABC) super family that functions specifically as a carrier mediated primary active efflux transporter. It is widely distributed throughout the body and has a diverse range of substrates that include several vital therapeutic agents whose bioavailability is reduced or resistance is induced because of the protein efflux. P-gp was first discovered in 1976 for its role in multi-drug resistance in cancer; it is over expressed in several human tumors and is an important barrier to success in cancer treatment. Due to its role in drug metabolism, P-gp has considerable clinical relevance as it affects the absorption, distribution and secretion of anticancer drugs [1]. Several natural products from plants and marine sources have been reported for their P-gp inhibitory action and thereby serving as potential chemopreventives when co-administered with anti-cancer agents. The P-gp inhibitory compounds from plant sources belong to various categories such as flavonoids, stilbenes, coumarins, terpenoids, alkaloids and saponins whereas the P-gp inhibitors from marine natural products belong to the class of polyketides.

Several flavonoids have been reported to evince P-gp inhibition such as flavonoids from grape fruit juice such as kaempferol and naringenin, flavonoid from Scutellariae radix viz. baicalein, procyanidine from bark of Pinus massoniana etc. P-gp inhibitors from other categories include resveratrol, a well-known stilbene, and coumarins from roots of Angelica gigas, dihydroxybergamotin and other furanocoumarins contained in grapefruit juice such as bergamotin, bergaptol and bergapten. Examples of alkaloids include glaucine, cepharanthine etc., example of diterpenes include euphomelliferine, and examples of steroidal saponins include primulanin, astragaloside II and karavelagenin C [2]. A number of P-gp inhibitors have been obtained from marine sources such as ecteinascidin 743 isolated from Caribbean tunicate Ecteinascidia turbidata, discodermolide, a polyketides from sponge Discodermia dissoluta, bryostatin 1 from bryozoans Bugulia neritina etc. [3]. These P-gp inhibitors from natural sources may act as potential enhancers of bioavailability of various anticancer drugs by preventing the multi drug resistance, reducing the effective dose of anticancer drugs and their associated side effects.

\section{References}

1. Srivalli KMR, Lakshmi PK (2012) Overview of P-glycoprotein inhibitors: a rational outlook. Brazillian Journal of Pharmaceutical Sciences 48: 353-367.

2. Abdallah HM, Al-Abd AM, El-Dine RS, El-Halawany AM (2015) P-glycoprotein inhibitors of natural origin as potential tumor chemo-sentitizers: a review. Journal of Advanced Research 6: 45-62.

3. Lopez D, Martinez-Luis S (2014) Marine natural products with P-glycoprotein inhibitor properties. Marine Drugs 12: 525-546.
*Corresponding author: Dr. Pankaj Gupta, Assistant Professor, School of Medical and Allied Sciences, KR Mangalam University, Sohna Road, Gurgaon-122 103, Haryana, India, Tel: +91119818256122; E-mail: pankaj.gupta@krmangalam.edu.in

Received May 30, 2016; Accepted May 31, 2016; Published June 04, 2016

Citation: Gupta P (2016) P-Glycoprotein Inhibitors from Natural Sources as Chemopreventives. Nat Prod Chem Res 4: e116. doi:10.4172/2329-6836.1000e116

Copyright: (C) 2016 Gupta P. This is an open-access article distributed under the terms of the Creative Commons Attribution License, which permits unrestricted use, distribution, and reproduction in any medium, provided the original author and source are credited. 